\title{
Validation of a rodent model of episodic memory
}

\author{
Wenyi Zhou and \\ University of Georgia, Athens, GA, USA \\ Jonathon D. Crystal \\ University of Georgia, Athens, GA, USA. Department of Psychological and Brain Sciences, \\ Indiana University, Bloomington, IN 47405, USA
}

Jonathon D. Crystal: jcrystal@indiana.edu

\begin{abstract}
Episodic memory consists of representations of specific episodes that happened in the past. Modeling episodic memory in animals requires careful examination of alternative explanations of performance. Putative evidence of episodic-like memory may be based on encoding failure or expectations derived from well-learned semantic rules. In Experiment 1, rats were tested in a radial maze with study and test phases separated by a retention interval. The replenishment of chocolate (at its study-phase location) depended on two factors: time of day (morning vs. afternoon) and the presence or absence of chocolate pellets at the start of the test phase. Because replenishment could not be decoded until the test phase, rats were required to encode the study episode. Success in this task rules out encoding failure. In Experiment 2, two identical mazes in different rooms were used. Chocolate replenishment was trained in one room, and then they were asked to report about a recent event in a different room, where they had no expectation that the memory assessment would occur. Rats successfully answered the unexpected question, ruling out use of expectations derived from well-learned semantic rules. Our behavioral methods for modeling episodic memory may have broad application for assessments of genetic, neuroanatomical, neurochemical, and neurophysiological bases of both episodic memory and memory disorders such as those that occur in Alzheimer's disease.
\end{abstract}

\section{Keywords \\ Episodic memory; Episodic-like memory; What-where-when memory; Encoding; Unexpected question; Rats}

\section{Introduction}

Episodic memory is defined as a recollection of an event that occurred at a particular time and place in the past (Tulving 1983, 2002). There has been an ongoing debate as to whether episodic memory is a uniquely human ability (Roberts et al. 2008; Tulving and Markowitsch 1998; Tulving 1972, 1983). Because of the inability to evaluate subjective experiences and autonoetic consciousness (Tulving 2002), researchers have instead focused on the content of episodic memory, often referred to as episodic-like memory or what-where-when memory, in a number of non-human species, such as scrub jays (Clayton et al. 2003a; Clayton and Dickinson 1998, 1999a, c), rats (Babb and Crystal 2005, 2006a, b; Zhou and Crystal 2009),

Correspondence to: Jonathon D. Crystal, jcrystal@indiana.edu.

Conflict of interest The experiments complied with the current laws of the country in which they were performed. The authors declare that they have no conflict of interest. 
pigeons (Zentall et al. 2008; Singer and Zentall 2007; Zentall et al. 2001), magpies (Zinkivskay et al. 2009), chickadees (Feeney et al. 2009), and monkeys (Hoffman et al. 2009). The development of animal models of episodic memory holds enormous potential for studying the underlying mechanisms of human memory processes and the biological basis of memory pathologies. However, two major concerns have been identified in recent approaches that model episodic memory in rats. The putative evidence for episodic-like memory may be explained by two non-episodic-memory solutions: (1) failing to encode a specific episode (the encoding failure hypothesis) (Zhou and Crystal 2009) and (2) using expectations generated from well-learned semantic rules that may develop with extensive training (Zentall et al. 2008, 2001; Singer and Zentall 2007). Our studies aimed to address these two concerns. We begin with a brief review of the relevant literature that leads to these concerns.

\section{A brief review}

Clayton and Dickinson (1998) developed behavioral criteria for studying episodic memory in animals based upon the three properties of an episode: what (the specific event) had happened, when and where a unique event occurred (Tulving 1972). Their studies have shown that scrub jays have a detailed representation of what (worm or peanuts) had occurred, where (location in the caching tray) and when (the retention interval between caching and recovering) the episode occurred (de Kort et al. 2005; Clayton and Dickinson 1998, 1999a, b, c; Clayton et al. 2001; Clayton et al. 2003b). Adapting Clayton and Dickson's paradigm, Babb and Crystal (2005, 2006a, b) demonstrated what-where-when memory in rats. In the study phase, rats were first exposed to an 8-arm radial maze with food accessible in four of the arms. One of the arms contained distinctively chocolate-flavored pellets, and the other three contained regular chow-flavored pellets. After either a short (e.g., $1 \mathrm{~h}$ ) or a long (e.g., $4 \mathrm{~h}$ ) retention interval, rats were put back in the maze with all arms opened for a test phase. Chow was only available at the arms that were previously inaccessible. The arm that had initially contained chocolate pellets was replenished after a long retention interval, but not after a short retention interval. Rats revisited the distinctive location more frequently after the long retention interval than after the short retention interval, suggesting memory of what (chow vs. chocolate pellets), where (the location baited with chocolate), and when (different retention intervals).

Although rats demonstrated what-where-when memory in this study, one potential criticism is that these rats might have adopted different strategies based upon time of day (Hampton et al. 2005). Instead of remembering the content of a specific episode, rats might have been using a set of rules to revisit the chocolate location at different rates in the morning vs. afternoon, thereby providing a non-episodic solution to the task. In a later study, Babb and Crystal (2006a) controlled time of day by using a short retention interval of $1 \mathrm{~h}$ and a long retention interval of $25 \mathrm{~h}$. Because the study occurred at a constant time of day, the circadian representation of the time of day at test could not be used as a cue to select different search strategies at different times of day. When chocolate was replenished after $25 \mathrm{~h}$, rats revisited the chocolate location at a higher rate after the long than after the short retention interval. These results suggest that the discrimination of what, where, and when was not based on adopting different search strategies at different times of day at test. Moreover, when chocolate was devaluated by pairing it with lithium chloride $(\mathrm{LiCl})$ after encoding whatwhere-when information, the rats reduced revisits to the chocolate location. These data suggest that knowledge of what-where-when was flexibly updated when new information was provided.

It has been argued that in the studies described above, the rats could have timed different retention intervals (i.e., use of a how-long-ago cue) rather than remembering features of an 
earlier event including when the event occurred (Roberts et al. 2008). Importantly, the relative familiarity of encoded events would be different after short and long retention intervals, thereby providing a non-episodic solution to the task. Roberts and his colleagues (2008) elegantly unconfounded these two variables (i.e., how long ago and absolute memory of when). For rats in the "how long ago" group, the reward contingency was based upon the retention interval between the study and test phases; for rats in the "when" group, the reward contingency was based upon the study time of day. The "how long ago" group learned to discriminate what, where, and when, but the "when" group did not. Roberts et al. (2008) concluded that rats timed the retention interval since the event occurred without remembering "when" the earlier event occurred. These data suggest that when both "when" and "how-long-ago" cues are relevant to predicting replenishment, the how-long-ago cue appears to dominate.

Zhou and Crystal (2009) tested for memory of "when" while controlling relative familiarity of events. They used a constant retention interval in all trials to eliminate the usefulness of the how-long-ago cue. Testing occurred either in the morning or in the afternoon, and chocolate replenished at one of these times of day; thus, time of day was the only available temporal cue that could be used to predict replenishment of chocolate. Rats learned the discrimination, indicating they were capable of remembering the absolute time of day at which an earlier event occurred, under conditions in which how-long-ago cues were uninformative. When the role of time of day at the study phase and time of day at the test phase were dissociated, it was found that rats remembered the time of day at the study phase rather than using the time of day at the test phase. Therefore, they concluded that at the time of memory assessment, rats remember when an event occurred. These data are consistent with the hypothesis that the rats remembered a unique earlier event.

Zhou and Crystal (2009) provided evidence that rats remembered when an event occurred, suggesting that rats use knowledge of what-where-when. However, it may be argued that the rats might have learned that encoding the chocolate location was not required in some timeof-day conditions, which we refer to as the encoding failure hypothesis. For example, a rat might solve the task by encoding the location of chocolate at one time of day (e.g., when chocolate replenishes in the morning), but not encoding the location of chocolate at the other time of day (e.g., when chocolate does not replenish in the afternoon); notice that differential rates of revisiting chocolate would occur in this situation without remembering the episode. The encoding failure hypothesis could also explain data from other studies. For example, using an approach similar to Babb and Crystal's (2005, 2006a, b) design, Naqshbandi et al. (2007) varied the time of day at the study while maintaining a constant time of day at the test (i.e., a short and long retention interval between the study and test phases). The rats showed evidence of what-where-when memory. However, it could also be argued that the rats failed to encode the event based upon information provided by time of day at the study phase.

It is not known if rats selectively encoded the location of chocolate in our earlier study (Zhou and Crystal 2009). However, if rats selectively encoded the chocolate location, they would have produced data like those reported by Zhou and Crystal without using episodic memory. It is important to note that our claim is not that the alternative hypothesis is likely or unlikely. Rather, we believe that the existence of an alternative explanation means that it would be premature to claim that the body of research documents episodic-like memory without testing the alternative hypothesis. Our project of validation requires careful elimination of this alternative explanation. Thus, we tested the encoding failure hypothesis in Experiment 1. 
Many episodic-like memory paradigms in animals have used extensive amounts of training, which may foster the development of well-learned expectations about future to-be-rewarded response(s) (Singer and Zentall 2007; Zentall 2005, 2006; Zentall et al. 2001, 2008). Importantly, putative episodic-like memory performance may be based on application of expectations derived from well-learned semantic rules. We address this concern in Experiment 2.

\section{Experiment 1a: encoding failure hypothesis}

We provided rats with daily information about a preferred food type (chocolate) that replenished or failed to replenish at its previously encountered location (Fig. 1); chowflavored food was available at all other locations, but did not replenish. Some of the information needed to predict replenishment was available at the time of encoding (location, time of day, food flavor). However, one critical piece of information needed to predict replenishment was not presented until immediately before the memory assessment; the presence or absence of additional chocolate pellets in a central location could be used to predict replenishment when combined with time of day. Importantly, although time of day was known at time of study, the subsequent baiting of the central location could not be predicted at time of study. Thus, to solve this task, it was necessary to encode the location of chocolate and time of day at study, but decoding of replenishment was delayed until immediately prior to memory assessment. To preferentially revisit the chocolate location when it was about to replenish at their second helpings of food (memory-assessment phase), the rats needed to remember where they found it during their first helpings of food (encoding phase) on that same day. For example, for some rats, the presence of chocolate in the hub in the morning (left columns of Fig. 1) and the absence of chocolate in the hub in the afternoon (right columns of Fig. 1) allowed the rat to predict the forthcoming replenishment of chocolate. For other rats, the role played by presence and absence of food in the hub was reversed to counterbalance assignment of conditions across the rats. Because it was impossible to predict whether chocolate would be replenished later, rats had to encode the episode on each study occasion. If the encoding failure hypothesis explained the results from previous studies, it would be impossible for rats to solve the current task. By contrast, if rats encoded detailed information about chocolate, they should show a significantly higher revisit rate to chocolate when it was about to replenish compared with when it was not about to replenish.

\section{Materials and methods}

Animals-Twenty male Long-Evans rats (Rattus norvegicus [Harlan]; 67 days old and 273 $\mathrm{g}$, on average, at the start of the experiment) were housed individually in a colony (light onset and offset at 6:00 a.m. and 6:00 p.m. Eastern Standard Time, respectively). Water was available ad lib, except during brief testing periods. Each rat was given 45-mg chow and chocolate pellets (F0165 and F0299, respectively; Bio-Serv) during daily experimental sessions and 15-20 g/day of 5001-Rodent-Diet (Lab Diet) after testing was completed. The experimental procedures were approved by the University of Georgia institutional animal care and use committee and followed the guidelines of the National Research Council Guide for the Care and Use of Laboratory Animals.

Apparatus-An 8-arm radial maze (described in Babb and Crystal 2005, 2006a) consisted of a central hub and 8 guillotine doors. A food trough and a 45-mg pellet dispenser were located at the distal end of each arm. A photobeam in the trough detected head entries. Other photobeams were 3.8 and $5.1 \mathrm{~cm}$ from guillotine doors. White noise masked outside noise. The experimental events (guillotine doors and food) were controlled by a computer in an adjacent room. Data (photobeam breaks, doors, food) were recorded (10-ms resolution) with 
MED-PC software (version 4.0). After each rat was removed from the maze, the maze was cleaned with Nolvasan (Fort Dodge Animal Health) throughout all procedures. Chow and chocolate pellets were placed beside each filled pellet dispenser (i.e., food odors were constant throughout all parts of the experiment). The maze was placed in a $3.50 \times 3.87 \mathrm{~m}$ room (which we refer to as Room 1), which was separated from the corridor by an adjacent room of approximately the same size. Arm 1 was oriented to the north. There was a bookshelf located on the north wall, a sink and a table on the east wall, and posters on the west wall. The entrance to the room was located on the north wall.

\section{Procedure}

Preliminary Training: Pretraining (3 20-min daily sessions) permitted the rats to explore the maze with one randomly selected arm, each day, baited with 3 chocolate pellets along the arm and a chocolate pellet in the food trough, and the remaining 7 arms each baited with chow in the same pattern. Initial training (20 daily sessions) permitted the rats to receive replenishment of chocolate-flavored food at one randomly selected daily arm and to earn chow-flavored food at each remaining arm. Daily sessions began at 7:30 a.m. for half of the rats and at 1:30 p.m. for remaining rats. Prior to placing the rats individually into the central hub, 15 chocolate pellets were placed in the hub for some rats, and the hub did not contain pellets for the remaining rats. At the start of each session, rats were individually placed in the central hub for $30 \mathrm{~s}$ during which they could eat the pellets (if present) and wait for the guillotine doors to open. In initial training, the arm baited with chocolate replenished as described below. Chow-baited arms never replenished. A visit was defined by the interruption of a food-trough photobeam; interruption of the photobeam near the guillotine door was required before the next interruption of a food-trough photobeam was counted as a visit. Each arm containing chow dispensed one chow pellet per day contingent on the first visit to each of these arms. The arm containing chocolate could dispense 3 chocolate pellets per visit. Rats could revisit arms with chocolate up to 5 times and receive 3 pellets per visit (additional food was not available after the fifth visit). In the first 10 sessions, pellets were placed in the hub, and subsets of rats were tested in the morning and afternoon conditions for which pellets predicted chocolate replenishment (see Fig. 1). In the next 10 sessions, the time of day at which the subsets were tested was reversed so that each animal was tested at the time of day at which the absence of pellets in the hub predicted chocolate replenishment. Daily sessions ended when food was earned at each arm or $10 \mathrm{~min}$ had elapsed.

Training: In the remainder of the procedure, each rat was placed in the maze twice per day (either in the morning [7:30 a.m.] or in the afternoon [1:30 p.m.], but not both) to provide an encoding opportunity (i.e., first helpings of food) followed by a memory assessment (i.e., second helpings of food). Each rat encountered chocolate at one and chow at three randomly selected radial-maze arms in a daily encoding phase. One pellet per arm was dispensed during first helpings of food. Next, the rat was removed, the maze was cleaned, and the hub was either baited or not baited with 15 chocolate pellets; these steps required $2.0 \pm 0.1 \mathrm{~min}$ (mean \pm SEM), on average, which constituted the retention interval. Next, the rat was placed in the hub, and after $30 \mathrm{~s}$, all guillotine doors were opened for a memory-assessment phase. Chow was available at previously inaccessible locations. The replenishment of chocolate (at its encoding-phase location) depended on two factors: time of day (morning vs. afternoon) and the presence or absence of chocolate pellets in the central hub at the start of the memory assessment. The assignment of time of day and hub-baiting combinations to replenishment and non-replenishment was counterbalanced across rats (half of the rats, randomly selected prior to the start of the experiment, had the conditions shown in Fig. 1, and the remaining rats had these conditions reversed). The memory-assessment phase ended when food had been dispensed at each of the baited locations (i.e., 4 or 5 different arms had provided food in non-replenish or replenish conditions, respectively). On any given day, only one session 
of replenishment or non-replenishment (but not both) was tested. Optimal performance is to revisit the chocolate location at the memory-assessment phase when replenishment is imminent but to reduce this tendency when chocolate replenishment is not forthcoming. Because chow-flavored locations from first helpings never replenished at second helpings, solving this task requires knowledge about what and where events occurred in addition to information about when the critical events occurred since chocolate was available at a dailyunique location that depended on time of day. Because the interval between study and test was constant within each experiment, how long ago a chocolate encounter occurred could not be used to predict replenishment. Training trial types were initially presented in consecutive blocks followed by presentation in a randomly mixed sequence, as described in the sections below. Because the replenishment or non-replenishment cue was not presented until after the retention interval, encoding of the chocolate location was required in each study phase.

Block testing: Initially, the replenishment condition (as defined by a combination of time of day and hub baiting) was held constant for consecutive days within 8 blocks of 5-20 sessions ( 95 sessions overall). Blocked presentation of sessions was designed to facilitate detection of the prevailing replenishment condition associated with time of day and hubbaiting conditions. Chocolate replenished in the initial block, and replenishment condition alternated in subsequent blocks in a random order of presentation.

Mixed testing: In mixed testing, replenishment and non-replenishment sessions (18 overall) were conducted in random order, using blocks of 6 sessions ( 3 sessions of each type), with the constraint that no more than 3 consecutive sessions of the same replenishment type occurred. In all other respects, mixed testing was the same as block testing.

\section{Data analysis}

The data come from sessions in which the time of day at which the session started was randomly mixed across days. The dependent measure was the probability of revisiting a location with a distinctive food type during the first four visits that occurred in the memoryassessment phase; the probability expected by chance (i.e., random arm entries) is 0.41 (calculated with a geometric distribution). For estimates of accuracy in avoiding chowflavored locations, a correct visit was defined as visiting an arm that was baited with chow in the memory-assessment phase, and the analysis of the first four choices was restricted to the seven non-chocolate arms; accuracy expected by chance (i.e., random arm entries) is 0.46 (calculated by enumerating all possible sequences of arm entries). Statistical tests were considered significant at an alpha level of 0.05 .

\section{Results and discussion}

If rats encoded the episode in every trial and decoded the replenishment of chocolate when presented with the central-hub baiting condition, then they should preferentially revisit the chocolate location when it was about to replenish. Rats were more likely to revisit the chocolate location in the replenishment conditions compared with the non-replenishment conditions (Fig. 2; $F_{1,19}=38.3, p<0.001$ ). Revisit probabilities were not statistically different for both retrieval cues $\left(F_{1,19}=4.0, p=0.06\right)$, and the effect of replenishment condition did not depend on the retrieval cue $\left(F_{1,19}=2.4, p=0.14\right)$. Differential rates of revisiting chocolate-flavored locations were accomplished while rats accurately avoided revisits to depleted chow-flavored locations $(0.78 \pm 0.03$; mean $\pm \mathrm{SEM})$.

The results of this experiment rule out the encoding failure hypothesis. The rats revisited the chocolate location when it was about to replenish relative to the non-replenishment condition. To successfully solve this task, rats had to encode the episode at the first helpings 
of food, because the critical information about whether or not chocolate would be replenished at the recently visited location was not available until the presence or absence of chocolate pellets in the central hub immediately before the memory assessment.

\section{Experiment 1b: long-term memory}

Experiment 1a ruled out the encoding failure hypothesis as an alternative explanation of performance in episodic-like memory tasks in rats. However, episodic memory is part of long-term memory (Tulving 1985). To capture this feature, it is important to use a relatively long retention interval between the study and test phase. In this experiment, we substituted an approximately 1-h delay for the 2-min delay for each of the four conditions shown in Fig. 1 , using the same rats. We did this with a transfer test. Rats consumed their first helpings of food at the usual times of day (as in Experiment 1a); however, the memory assessment occurred $1 \mathrm{~h}$ later. Rats with episodic-like memory should show similar results to those observed in Experiment 1a: higher revisiting rates to the recently visited chocolate location in the replenishment conditions compared with non-replenishment conditions. No additional training should be needed to document this performance (hence the use of a transfer-test design).

\section{Materials and methods}

Animals and apparatus-We used the same subjects and apparatus as in Experiment 1a.

Procedure-In the transfer test, encoding- and memory-assessment phases were identical to the mixed testing described in Experiment 1a except: (1) the retention interval was $56.7 \pm$ 0.6 min (mean \pm SEM), on average, and (2) each of the four trial types (as shown in Fig. 1) was presented once, with the order of presentation randomly selected. The encoding phase occurred at a time that was familiar to the rats from earlier training (i.e., beginning at 7:30 a.m. or 1:30 p.m.), but the memory assessment was conducted at unfamiliar times, approximately $1 \mathrm{~h}$ after encoding (i.e., 8:30 a.m. or 2:30 p.m.). Because the four trial types were each tested once in random order, the data were collected before the rats obtained feedback about replenishment in each of these new conditions.

\section{Results and discussion}

Rats revisited the chocolate location in the replenishment conditions at a higher rate than in the non-replenishment conditions (Fig. $3 ; F_{1,19}=8.4, p=0.009$ ). Revisit probabilities were similar for both retrieval cues $\left(F_{1,19}=1.0, p=0.33\right)$, and the effect of replenishment condition did not depend on the retrieval cue $\left(F_{1,19}=2.4, p=0.14\right)$. Differential rates of revisiting chocolate-flavored locations were accomplished while rats accurately avoided revisits to depleted chow-flavored locations $(0.68 \pm 0.03$; mean \pm SEM $)$. When tested at novel times after a longer delay, rats remembered what, where, and when the earlier event occurred.

In the present experiment, it is possible that the rats used time of day at study or at test as the when component of what-where-when memory. However, we believe that it is unlikely that the rats used time of day at test for three reasons. First, Zhou and Crystal (2009) conducted a transfer test in which a 7-h retention interval replaced a much shorter retention interval; on the very first trial with a familiar study time and a novel test time, the rats continued to revisit the chocolate location at the appropriate, differential rates. Thus, use of test time was ruled out in an experiment that used a very similar training procedure. Second, Zhou and Crystal conducted a conflict test in which the trial started with a familiar "afternoon" study time and ended with a familiar "morning" test time; in the conflict test, the rats used study time rather than test time to adjust revisit rates to chocolate. Thus, use of test time was ruled 
out again. Third, the present Experiment $1 \mathrm{~b}$ is quite similar to the transfer test of Zhou and Crystal as described above. The only difference between these two experiments is that the present experiment used a 1-h retention interval rather than the 7-h retention interval. In the present Experiment 1b, we also observed complete transfer, meaning that the rats appear to have responded based on the familiar study times rather than the unfamiliar test times. However, it is worth noting that the evidence is stronger in the 7-h test described above because a larger retention interval makes the test times more dissimilar than in earlier training.

\section{Experiment 2: unexpected question}

Our efforts to validate a rodent model of episodic memory focus on ruling out potential alternative explanations that do not require memory of a specific earlier episode. Zentall and colleagues (Singer and Zentall 2007; Zentall et al. 2001, 2008) have argued that extensive training in what-where-when memory experiments may promote the use of expectations derived from well-learned semantic rules; thus, validation of our rodent model requires elimination of this alternative. Zentall and colleagues have focused on the expectations that develop by virtue of the extensive training required by rule learning. They have argued that one potential solution to this problem is to evaluate an animal's ability to answer an unexpected question; when a question is unexpected, relative to earlier training, answering the question requires retrieval of the earlier episode. To develop this argument, we first note that when studying some information, there is a set of expectations about how that information will be used in the future. To ask an unexpected question, the memory assessment is conducted in a novel fashion (i.e., in a way that cannot be expected based on earlier training). The defining feature of an unexpected question is as follows: at the time of memory encoding, although there are a number of expectations about the test that may develop at study, some of the critical expectations are absent. An example from Zentall's experiments will be described next, followed by an analysis of what is expected and unexpected in this paradigm. Based on this analysis, we developed a test of an unexpected question for rats performing our radial-maze task.

In Zentall et al.'s (2001) study, the pigeons were initially trained in a symbolic matching to sample task with a required pecking (or not pecking) response inserted between sample and comparison phases of each trail. Based on extensive training with this procedure, we may assume that when the pigeons encoded the sample, they may have formed an expectation to choose the associated comparison color. Next, the pigeons were trained in a Pavlovian procedure in which yellow, but not blue, was associated with food. In the final test, the pigeons were presented with yellow or blue, which occasioned pecking or not pecking, and were then given the novel test condition in which the comparison choices were the colors from the symbolic matching to sample task. Although pecking, a moment earlier, was incidental to the presentation of yellow or blue, the pigeons were able to report that they had just pecked or refrained from pecking. Importantly, at the time of presentation of the Pavlovian cues and pecking, the pigeons could not have expected to receive a comparison of the choice keys from the symbolic matching to sample task. Thus, Zentall et al. argued that the pigeons answered an unexpected question. They further argued that the pigeons retrieved an episodic memory of the recent pecking or non-pecking to answer the unexpected question.

The goal of our task was to use two rooms to construct an unexpected question. In initial training (as in Experiment 1a), the rats learned the contingencies that predicted replenishment of chocolate in Room 1 . They were familiarized with a nearby room (Room 2 ), but they were never exposed to chocolate or its replenishment in Room 2. After observing that the rats can discriminate the two rooms, we used a probe test to assess 
performance with an unexpected question. The study phase with chocolate began in Room 1, as in previous training. We may assume that the rats encoded the location of chocolate within Room 1 and planned to revisit that location or not depending on the identity of the retrieval cue to be provided immediately prior to the test phase. Importantly, this would be an expectation to revisit the location in Room 1 defined by the global environmental cues in that room (e.g., Brown 1992; Brown et al. 1993; Mazmanian and Roberts 1983; Olton and Collison 1979; Roberts 1998; Suzuki et al. 1980). The trial continued with a test in Room 2. We used the retrieval cue that would prompt revisiting of the chocolate location. At the time of encoding in the probe, the rat is expected to store memories of Room 1 locations with respect to Room 1 global environmental cues. It is not possible for the rat to store information with respect to Room 2 cues at the time of encoding because the rat cannot know that it will be unexpectedly tested in Room 2. When the rat is tested in Room 2, it must retrieve a memory of Room 1 and use it in Room 2.

\section{Experiment 2a: two-room training-integration vs. segregation}

The objective of this experiment was to train rats to learn a set of experimental contingencies between two eight-arm radial mazes located in two different rooms. We used two identical radial mazes. The apparatus from Experiment 1 is referred to as Maze 1, and it was located in Room 1. The second maze is referred to Maze 2, and it was located in Room 2. Mazes 1 and 2 were positioned with the same orientation in rooms 1 and 2, and the arms in the two mazes were numbered in a corresponding sequence (i.e., arm 1 in Maze 1 was oriented the same direction as arm 1 in Maze 2). In each trial, rats received their first helpings of food in Maze 1 in Room 1. In the first helpings, four arms were randomly selected, and all arms were baited with regular chow pellets; note that chocolate was not used in Experiment 2a. After encountering the study phase, rats were brought back to the colony before being taken to Maze 2 in Room 2 for the second helpings of food. In second helpings, the trial continued with all eight arms open. Chow pellets were only baited at the four arms in Maze 2 that corresponded to those that were inaccessible earlier in first helpings in Maze 1 (i.e., the trial continued across the two rooms). Rats were given the opportunity to deplete the two helpings of chow in two different mazes in two rooms. If rats readily integrated events from the two rooms, then they would show a high level of accuracy in avoiding arms in Maze 2 that corresponded to the recently visited arms in Maze 1. If rats did not readily integrate events from the two rooms, then they would perform at a low level of accuracy in avoiding unbaited arms in Maze 2.

\section{Materials and methods}

Animals-Two rats died at the beginning of this experiment. The remaining 18 animals from Experiment 1 were used.

Apparatus-Both Maze 1 (described in Experiment 1a) and Maze 2 were used in this experiment. Maze 2 was identical to Maze 1. Maze 2 was located in Room 2. Room 2 was divided into two parts. The maze section $(3.54 \times 5.49 \mathrm{~m})$ was separated from the remainder of the room by three office dividing walls. Room 2 was accessible from the corridor. Maze 2 was placed in the center of the test space with Arm 1 oriented north. There was a sink located on the north wall, and a table was located on the east wall. No decorations were placed on any walls. The entrance to Room 2 was on the south wall. In summary, the rooms and the mazes, each had the same geographical orientation and locations of the table (on the east wall) with respect to the mazes. They differed in a number of respects, such as the size and decoration of the two rooms, locations of the sink and the entrance to the room. 


\section{Procedure}

Two-room training: Two-room training (33 daily sessions) provided rats an opportunity to learn that a trial that started in Room 1 continued in Room 2. Each rat was placed in the maze twice per day as in Experiment 1a and 1b except as follows: (1) the encoding opportunity (first helpings of food) and the memory assessment (second helpings of food) were conducted in two different rooms: first helpings of food occurred in Room 1, and second helpings of food occurred in Room 2; (2) only chow pellets were used (i.e., chocolate pellets were not presented in the current experiment); (3) each rat was returned to the colony after finishing the encoding phase in Maze 1, and next the rat was taken to Maze 2 for a memory-assessment phase; (4) the retention interval was $4.6 \pm 0.1$ min (mean \pm SEM), and (5) the central hub was never baited with food. In Room 1, each rat encountered chow at four randomly selected radial-maze arms in a daily encoding phase. Next, the rat was taken out of the maze and brought back to the colony before being brought to Room 2 for a memory-assessment phase. The time taken for this transition constituted the retention interval. In Room 2, the rat was put into Maze 2 for the memory assessment. Chow was available at four locations that were previously closed at the corresponding arms in Maze 1. In this experiment, the rats were always put into two mazes oriented between arms 3 and 4 . Overall, rats were given the opportunity to learn that trials that started in Room 1 continued in Room 2.

\section{Data analysis}

For estimates of accuracy in visiting chow-flavored locations in Maze 2, a correct visit was defined as visiting an arm that was baited with food, and the analysis of the first four choices included all eight arms; accuracy expected by chance (i.e., random arm entries) is 0.45 (calculated by enumerating all possible sequences of arm entries).

\section{Results and discussion}

The accuracy of avoiding chow arms in Maze 2 that corresponded to recently visited arms in Maze 1 was $0.51 \pm 0.01$ (Mean \pm SEM). Although this level of performance was reliably above the rate expected by chance $\left(t_{17}=6.2, p=0.00001\right)$, the magnitude of the difference was quite small. Indeed, Room 2 performance involved avoiding arms that were baited in Room 1 at a rate of 0.06 , which corresponds to avoiding approximately 1 previously baited arm out of 20 arm choices. Moreover, there was no evidence for improvement over sessions in any rat. In conclusion, rats do not readily integrate events across two rooms. Clearly, the rats discriminated the two rooms.

\section{Experiment 2b: unexpected question with chocolate}

In this experiment, we assessed rats' ability to answer an unexpected question. First, we retrained rats on two of the conditions from Experiment 1 (Fig. 1) in Room 1. One replenishment condition was predicted by the presence of chocolate pellets in the central hub, and one non-replenishment condition was predicated by the absence of chocolate pellets in the central hub. Next, after rats showed differential rates of revisiting in the two conditions, we conducted a probe test. In the probe test, a chocolate pellet was provided in one of the arms in Room 1, and later chocolate pellets were provided in the central hub in Room 2, which predicted chocolate replenishment. Chocolate replenishment occurred in the arm corresponding to the chocolate-baited arm in Room 1 (i.e., the trial continued in Room 2 using contingencies originally established in Room 1). Importantly, the rats had never encountered chocolate in Room 2 prior to the probe. Consequently, it may be argued that, at the time of encoding chocolate in Room 1, the rats could not have expected that chocolate would replenish in Room 2 . They were unexpectedly asked to retrieve information about the expected location of chocolate in a novel context. If rats can answer this unexpected 
question, then they should revisit the corresponding chocolate location in Room 2 at a relatively high rate on the first trial in which the unexpected question occurred.

\section{Materials and methods}

Animals and apparatus-The rats and apparatus were the same as Experiment 2a.

\section{Procedure}

Training: Two conditions from the design of Experiment 1 were used. These two conditions were the replenishment condition combined with the presence of 15 chocolate pellets in the central hub and the non-replenishment condition combined with the absence of chocolate pellets; because of the original counterbalance assignment from Experiment 1, the two conditions were conducted in the morning for half of the animals and in the afternoon for the remaining animals. In each session, rats were returned to the colony after the encoding phase in Room 1 and then taken back to Room 1 for a memory-assessment phase. The retention interval was $3.7 \pm 0.1$ min (mean \pm SEM). In the initial mixed testing, replenishment and non-replenishment conditions (overall 14 sessions) were conducted in random order. Next, rats were presented with a block of non-replenishment trials (16 sessions) to reduce the rate of revisiting chocolate. Finally, rats received 10 sessions of mixed testing, which served as the baseline for replenishment and non-replenishment conditions.

Refresher training: Two trials of the two-room training with chow pellets only were conducted before the probe test. These trials were identical to the trials described in Experiment $2 \mathrm{a}$ and were designed to eliminate the relative novelty of being tested in two rooms.

Probe test: In the probe test, the initial encoding phase was conducted in Room 1. Rats encountered chocolate at one and chow at three randomly selected arms in Maze 1. Rats were taken to the colony and then to Room 2 during the retention interval of $4.3 \pm 0.3 \mathrm{~min}$ (mean \pm SEM). Instead of continuing the trial in the same room, the trial continued in Room 2. In Room 2, 15 chocolate pellets were placed in the central hub to prompt the rats to retrieve the study episode. The chocolate was replenished at the Maze 2 arm that had the corresponding orientation to the arm in Maze 1 that had recently provided chocolate. Because chocolate had never appeared in Room 2, rats could not have expected to have chocolate replenish in Room 2. Thus, the test was unexpected. If rats can answer the unexpected question, they would show a relatively high rate of revisiting the corresponding chocolate location in Maze 2. By contrast, if rats cannot answer the unexpected question, rats would show no difference in the revisit rate to the chocolate location in Maze 2 compared to the baseline measure.

\section{Results and discussion}

Two baseline measures were used to evaluate performance in the probe test. The first baseline measure was the revisit rates to the chocolate location in both replenishment and non-replenishment conditions. It was obtained from performance in the last 10 sessions of mixed testing; we will refer to these data as the mixed baseline. Rats revisited the chocolate location at a higher rate in the replenishment condition compared with the nonreplenishment condition (Fig. $4 \mathrm{a} ; t_{17}=2.50, p=0.02$ ). The second baseline measure was the probability of visiting one randomly selected arm in Maze 2; we will refer to these data as the designated baseline. It was obtained from randomly assigning one arm in each session of the two-room training from Experiment 2a as a "designated distinctive" arm; this is the probability of revisiting a randomly designated arm in Maze 2 when there were no physical 
features to distinguish this arm from other arms. Note that there was no chocolate in the arm; the purpose of designating an arm in this analysis was to estimate a rate of entry of one randomly selected arm. The designated baseline was 0.49 (and it is labeled as Designated in Fig. 4b, c).

Rats showed a significantly higher revisit rate to the corresponding chocolate location in Maze 2 when chocolate was unexpectedly replenished (labeled as Chocolate Probe in Fig. $4 \mathrm{~b})$ compared with the non-replenishment mixed baseline in Maze $1\left(t_{17}=3.26, p=0.005\right)$. Chocolate probe performance was not significantly different from the replenishment mixed baseline condition (Fig. $4 \mathrm{~b} ; t_{17}=0.66, p=0.52$ ). When one of eight arms in each session of two-room training in Experiment 2a was randomly designated as a distinctive arm, it was found that rats were more likely to visit the chocolate location in Maze 2 in the probe test compared to the designated arm (Fig. $4 \mathrm{~b} ; t_{17}=3.81, p=0.001$ ). Differential rates of revisiting the chocolate-flavored location were accomplished while rats moderately avoided revisits to depleted chow-flavored locations $(0.60 \pm 0.04$, mean \pm SEM $)$.

Discussion of alternative explanations will be deferred until the discussion of Experiment 2c.

\section{Experiment 2c: unexpected question with sucrose}

Because rats had experienced chocolate in Maze 1 during previous training, chocolate was not novel to the rats. Consequently, one may argue that a retrieval test for memory of chocolate in a novel context may not be "unexpected." Therefore, it is important to document that rats are able to report about an earlier study event when the study event was novel in addition to an unexpected request to retrieve this information. In Experiment 2c, we replaced chocolate with a novel flavor, sucrose. If rats are able to report about the earlier event of a novel flavor in a novel context, we would expect a relatively high revisit rate to the corresponding arm in Maze 2 that was recently baited with sucrose in a different room. If rats were not able to report the earlier experience of a novel flavor at a novel context, we would not expect to obtain a relatively high revisit rate to the corresponding sucrose arm.

\section{Materials and methods}

Animals and apparatus-Animals and apparatus were the same as Experiment $2 \mathrm{~b}$.

Procedure-One trial of the probe test with sucrose was conducted. The procedure was the same as Experiment 2b, except that sucrose was used instead of chocolate in one of the eight arms. Sucrose replaced chocolate in the distinctively baited arm in the study phase (Room 1) and test phase (Room 2). The hub was baited with chocolate pellets (Room 2) immediately prior to the start of the test phase.

\section{Results and discussion}

Rats visited the sucrose location at a significantly higher rate when rats were unexpectedly asked to retrieve the memory of sucrose in Maze 2 (labeled as Sucrose Probe in Fig. 4c) compared with the non-replenishment mixed baseline (Fig. $4 \mathrm{c} ; t_{17}=1.94, p=0.03$, onetailed); use of a one-tailed test is appropriate in this case because the only prediction of any theoretical interest is a higher rate of revisiting sucrose in this replenishment condition. The sucrose probe revisit rate was not significantly different from the replenishment mixed baseline (Fig. $4 \mathrm{c} ; t_{17}=0.21, p=0.84$ ). Rats were more likely to visit the sucrose location in Maze 2 in the probe test compared to the designated baseline (Fig. $4 \mathrm{c} ; t_{17}=2.74, p=0.01$, two-tailed). Differential rates of revisiting the sucrose flavored location were accomplished while rats moderately avoided revisits to depleted chow-flavored locations $(0.58 \pm 0.05$, 
mean \pm SEM). In this experiment, rats had no expectation of encountering a novel flavor; nor did they have any expectation that they would be asked to retrieve a memory of sucrose in a different maze. Rats retrieved a memory of an earlier episode when they were unexpectedly asked to do so.

Performance in the probes of Experiment $2 b$ and $2 c$ is surprisingly good given the relatively poor performance in the two-room training of Experiment 2a. To explore the source of this improvement, we sought to determine whether the improvement was present from the beginning of the probe test phase. Thus, we examined accuracy on the first choice of test phases in the probes (combining across Experiments $2 b$ and $2 c$ ) and compared it to the expected level of performance from the two-room training (Experiment 2a). Accuracy in selecting a baited location was higher on the first choice in the probes $(0.67 \pm 0.08$, mean \pm SEM) compared to first choice performance in Experiment $2 \mathrm{a}(0.49 \pm 0.02$, mean $\pm \mathrm{SEM})$, and this difference was significant $\left(t_{17}=2.26, p=0.037\right)$. We propose that chocolate in the hub during the probes served as a cue to retrieve memory of Room 1 , which facilitated accurate performance in Room 2.

In Experiments $2 \mathrm{~b}$ and $2 \mathrm{c}$, we examined the ability of rats to use information in a novel context. When chocolate or sucrose was encountered in Room 1 (Experiments $2 \mathrm{~b}$ and 2c, respectively), we may assume that the rats encoded its location with respect to global environmental cues in Room 1. Any application of rule learning could lead the rats to expect to return to the distinctively baited location in Room 1 when given the opportunity to do so at the subsequent test phase. However, in our probe tests, the test phase was conducted in Room 2. In each probe test, the rats revisited the arm in Room 2 that corresponded to the Room-1 replenishment arm at the rate expected in the replenishment condition. In a very similar situation (Experiment 2a), the rats searched in a nearly random fashion when a trial started in Room 1 and continued in Room 2, suggesting that the rats discriminate the two rooms. Thus, successful completion of the trial in Experiment $2 \mathrm{~b}$ cannot be based on an inability to discriminate the two rooms. In Room 2, we conducted a single probe test with chocolate and a single probe test with sucrose. Moreover, none of the earlier training in Room 2 (Experiments 2a and 2b) involved chocolate or replenishment. Thus, the observed high revisit rates in the probe tests were not based on learning the consequences of visiting the corresponding location in Room 2; we used probe tests so that our data were collected before the rats had an opportunity to learn about the new condition.

There are two new features that were introduced in Experiment 2b: first, chocolate was presented in the study phase, and second the central hub was baited immediately prior to the test phase. Presentation of a chocolate-baited arm in the study phase may have generated an expectation that chocolate may replenish, and presentation of chocolate in the hub may have functioned as a retrieval cue for the study information. Either of these features may have contributed to the ability of the rats to integrate across the two rooms in Experiment $2 \mathrm{~b}$. Importantly, prior to our probe tests, application of well-established rules would generate solutions with respect to cues in Room 1 rather than solutions with respect to cues in Room 2 .

An additional new feature was introduced in Experiment 2c. The distinctively baited location in Room 1 had sucrose rather than chocolate. Although the rats had extensive experience with chocolate-baited locations replenishing, sucrose-baiting was novel in the probe test in Experiment 2c. It is possible that the rats coded the distinctively baited locations throughout these studies as "distinctive" or "yummy" etc. rather than coding specific flavors. However, we believe that it is unlikely that chocolate and sucrose locations are coded in this non-specific fashion. Babb and Crystal (2006b) conducted a number of content-selectivity tests of what-where-when memory. In one test, rats were trained to revisit 
or refrain from revisiting grape and raspberry locations depending on a temporal component. At the time of encoding the locations of these flavors, both grape and raspberry were highly valued food types. During the retention interval, the value of one (but not the other) flavor was reduced by giving the rats access to a large amount of one (but not the other) flavor. This flavor-specific satiation test revealed that the rats flexibly and selectively reduced revisits to the devalued flavor. In a second test, rats were trained to revisit or refrain from revisiting chocolate and banana locations. After encoding the location of these flavors, chocolate was paired with $\mathrm{LiCl}$ to produce a flavor-specific devaluation. When the trial continued, every rat revisited the banana location, and visits to the chocolate location were virtually eliminated. These findings suggest that the rats have a detailed representation of the flavors encountered in what-where-when memory. Thus, if the rats generalized the rule about chocolate in Experiment 2c, it was not because they had encoded the location in a non-specific fashion. Consequently, revisiting the location that corresponded to sucrose in Experiment $2 \mathrm{c}$ represents an additional novel context for assessing the ability of rats to answer an unexpected question.

One potential strategy that the rats may have used in the probe tests is dead reckoning (Gallistel 1990; Wallace et al. 2002, 2008). Indeed, the correspondence between Rooms 1 and 2 was defined by the compass orientation of each maze. However, it is noteworthy that dead reckoning alone does not explain our findings. In particular, if continuation of a trial in a different room was solved by dead reckoning alone, then the rats should have readily integrated across rooms in Experiment 2a. The virtually complete disruption observed in Experiment 2a suggests that the rats were using distinctive features of the room at study and test; when these features were different in study (Room 1) and test (Room 2) in Experiment $2 \mathrm{a}$, the rats appeared to be unable to integrate across rooms despite the availability of a dead reckoning solution. In Experiments $2 \mathrm{~b}$ and $2 \mathrm{c}$, the rats showed their ability to integrate across rooms to find the location that corresponded to the distinctively baited location in each probe test.

It is noteworthy that our probe is unlike the following analogy. Suppose that an undergraduate arrives to take a midterm exam and learns that it will be moved from room 101 to room 102. Indeed, in this case, there is no sense in which the midterm is unexpected because the content of the midterm is not about the room. However, when the rat encounters chocolate or sucrose in the study phase in Room 1, the rat encodes the study locations with respect to the global environmental cues in Room 1 (Brown 1992; Brown et al. 1993; Mazmanian and Roberts 1983; Olton and Collison 1979; Roberts 1998; Suzuki et al. 1980). Thus, the global environmental cues in Room 1 are part of the content of the hypothesized episodic memory. Importantly, at the time of study, it is not possible for the rat to form an expectation to navigate with respect to Room-2 global environmental cues. Consequently, moving to a new room with different global environmental cues represents a test about the content of the episode rather than merely a test in a new context. Documenting memory of the content of the episode makes this a model of episodic memory.

It is important to note that although the rats could not have expected to be tested in Room 2, they would expect to be tested in Room 1 . We recognize that this is a limitation to the sense in which the question is unexpected. However, our test has the following features of unexpectedness: The expectation of some specific details about the content of the test is not available at the time of study, despite the presence of other expectations at the time of study. The rats could have expected to revisit the chocolate location based on global environmental cues located in Room 1, but they could not have expected to visit a location with respect to global environmental cues in Room 2. When confronted with the retrieval cue (i.e., chocolate in the hub) in the probe, we propose that the rat retrieved a memory of Room 1, and it integrated (Blaisdell and Cook 2005; Chamizo et al. 2006; Cheng et al. 2007) 
representations of global environmental cues of the two rooms for the first time. The rat could not have known at the time of study that the integration would be required at the subsequent test. Yet, we believe that future research should use other methods to assess an unexpected question with reduced expectations at the time of study.

People remember episodes that were of limited importance when encoded. For example, if you ask someone what was the color of a building that was passed on the way to work, the person may be able to answer this unexpected question. Indeed, answering the question means that the information was encoded, but it may have been incidental and not apparently important at the time of encoding. The studies by Zentall and colleagues (Singer and Zentall 2007; Zentall et al. 2001, 2008) have this incidental feature: the keypeck occurs because of previous Pavlovian training but is not a to-be-studied piece of information when the peck occurs. In a later study, Zentall et al. (2008) refined their approach to more clearly document incidental encoding. A stimulus to-be-categorized with respect to orientation was presented on a left or right side, and the pigeons subsequently were asked to report on which side the stimulus had incidentally been presented earlier. By contrast, incidental encoding in our procedure is limited. When the rat encountered chocolate in Experiment $2 \mathrm{~b}$, it had extensive experience encoding that information for subsequent use (in Room 1). Importantly, although the information was not incidental at the time of study, the question was novel at the time of test (in Room 2). Because successfully finding the corresponding location in Experiments 2b and $2 \mathrm{c}$ cannot be based on an inability to discriminate Rooms 1 and 2 (based on Experiment $2 \mathrm{a}$ ), we argue that the rats retrieved a memory of Room- 1 information when tested in Room 2. In Experiment 2c, sucrose was presented for the first time, which may function to make its encoding more incidental (i.e., the rats did not have any previous history of encoding the location of sucrose for subsequent use). Nevertheless, it would be valuable to demonstrate that rats can answer an unexpected question when the information required was incidentally encoded using other methods in future research.

\section{General discussion}

The present experiments attempted to validate a rodent model of episodic memory by providing convergent lines of evidence (Crystal 2009, 2010; Shettleworth 1998). Two issues were addressed. First, our findings rule out the encoding failure hypothesis, suggesting that failing to encode the content of an episode is not used as an alternative strategy to solve what-where-when tasks. Second, rats demonstrated the ability to retrieve a memory of an earlier episode when they were unexpectedly asked to do so. The ability to answer an "unexpected" question captures a feature of human episodic memory—at the time of memory retrieval, information is used that could not be anticipated at an earlier point. Importantly, in our study, answering an unexpected question rules out the use of expectations derived from well-learned semantic rules established by extensive training.

The ability to find chocolate and sucrose in a novel context in the probes (Experiments $2 \mathrm{~b}$ and 2c) may also be interpreted as evidence of flexible use of memory of the study episode. It is noteworthy that the flexibility was likely afforded by the rats storage of a memory of the study episode rather than by storing the future response at the time of study. Documenting flexible use of study-episode memory is an important feature of episodic-like memory (Clayton et al. 2003a).

In an earlier study (Zhou and Crystal 2009), we speculated that it was unlikely that rats solved what-where-when memory tasks by selectively encoding the chocolate location when it could be predicted at study that it would be subsequently replenished at test. Here, we provide a definitive test of this non-episodic memory alternative hypothesis. Because replenishment could not be predicted until immediately before the memory assessment, rats 
could not use a differential encoding strategy (i.e., encode or fail to encode the chocolate location). Nevertheless, the rats solved the what-where-when task. Indeed, conditions at encoding were constant in the morning and afternoon, which each culminated in chocolate replenishment in $50 \%$ of the memory assessments, and did not become differentiated until immediately before the memory assessment. Thus, encoding was always required in this task. It is also noteworthy that the delay between encoding and memory assessment was constant in each experiment. Thus, the relative familiarity of earlier events was also constant and could not serve as a cue for predicting chocolate replenishment. In the study by Zhou and Crystal, rats continued to perform at above-chance levels in a transfer test with a 7-h retention interval. On the very first trial in these new conditions (i.e., before receiving any feedback about replenishment in the new conditions), the rats immediately continued to differentially revisit the chocolate location. This observation suggests that, at memory assessment, the rats retrieved information about when the study episode occurred, in addition to information about what happened and where the event occurred.

In the present experiments, we arranged for the presence or absence of 15 chocolate pellets in the central hub to serve as a retrieval cue. The present experiments suggest that these conditions are sufficient to promote their use in our task. Indeed, the presence of the chocolate pellets in the central hub in Experiments $2 \mathrm{~b}$ and $2 \mathrm{c}$ may have prompted retrieval of the Room-1 study episode, whereas the absence of pellets in the hub in Experiment 2a may have contributed to the apparent inability to integrate across rooms. However, it is not known if the use of a relatively large number of highly preferred food is necessary for these cues to be effective. It is noteworthy that we counterbalanced the role of chocolate in the hub so that the presence and absence of chocolate in the hub predicted replenishment of a chocolate arm for an equal number of rats. The presence of the chocolate-baited hub appeared to be more, though not significantly, effective at curtailing revisits to the nonreplenishing chocolate arm (Fig. 2), which may be related to flavor-specific satiation (Babb and Crystal 2006b); however, this apparent difference is absent in Fig. 3. It is well known that occasion setters have a profile of associative properties (Bouton 1997); however, the occasion setting properties in our task have not been explored. One approach to explore these issues is to use other putative occasion setting cues that are not appetitive (e.g., floor texture) in future studies.

The ability to recollect a unique past experience is a defining feature of episodic memory. A number of studies in animals have attempted to preserve this feature by performing a test in which animals have no prior exposure to the target event until the critical test (e.g., Dere et al. 2005; Zentall et al. 2008). The purpose is to prevent animals from developing semantic knowledge. In one of these approaches, when an animal's memory of a specific event is assessed, it has no expectation that it would be asked to report about that event. In the studies by Zentall and his colleagues (Zentall et al. 2001, 2008; Singer and Zentall 2007), pigeons demonstrated an ability to retrieve a recently encoded episode when their memory was unexpectedly assessed. Here, we document that rats retrieve an episodic memory when they are subsequently unexpectedly asked to do so. It is important to note that training with the distinctive flavor was only conducted in Room 1. In the probe test, rats were exposed to a distinctive flavor in Room 1, but they were unexpectedly asked to report the corresponding location based on different global environmental cues in Room 2. Successful performance in this task cannot be explained by expectations that developed through past training because rats had never encountered the distinctive flavor in Room 2. Thus, they should have no expectation of obtaining the distinctive flavor in this room based on different environmental cues. We increased the "unexpectedness" by using a novel flavor that had no training history. When rats were asked to retrieve a memory of the episode, they successfully answered the unexpected question. 
Our behavioral methods for validating a rodent model of episodic memory in rats may have broad application for assessments of the neurobiological bases of both episodic memory and memory disorders such as those that occur in Alzheimer's disease (Bäckman et al. 1999; Egerhazi et al. 2007; Kessels et al. 2007; Le Moal et al. 1997; Liscic et al. 2007; Nyberg et al. 1996). Several animal models of Alzheimer's disease mimic neurochemical and neuroanatomical phenotypes associated with Alzheimer's (Eriksen and Janus 2007; Oddo et al. 2003a, b; Yoshiyama et al. 2007). However, existing models typically evaluate more general measures of learning and memory (Watanabe et al. 2009; Volianskis et al. 2010; e.g., Blanchard et al. 2009; Christensen et al. 2010; Lovasic et al. 2005; Savonenko et al. 2005). Therefore, it is possible that therapeutic interventions that facilitate recovery of more general measures of learning and memory in animal models may leave profound episodicmemory impairments in clinical populations unimproved. Potential future applications of our behavioral assessment of episodic memory include RNA interference (Maxwell 2009) and targeted gene expression (Ueberham et al. 2006; Eriksen and Janus 2007; Hwang et al. 2004; Jankowsky et al. 2005; Keri et al. 2009).

\section{Acknowledgments}

This work was supported by National Institute of Mental Health Grant R01 MH080052 (to J.D.C.).

\section{References}

Babb SJ, Crystal JD. Discrimination of what, when, and where: implications for episodic-like memory in rats. Learn Motiv. 2005; 36(2):177-189.

Babb SJ, Crystal JD. Discrimination of what, when, and where is not based on time of day. Learn Behav. 2006a; 34(2):124-130. [PubMed: 16933798]

Babb SJ, Crystal JD. Episodic-like memory in the rat. Curr Biol. 2006b; 16(13):1317-1321. [PubMed: 16824919]

Bäckman L, Andersson JL, Nyberg L, Winblad B, Nordberg A, Almkvist O. Brain regions associated with episodic retrieval in normal aging and Alzheimer's disease. Neurology. 1999; 52(9):18611870. [PubMed: 10371535]

Blaisdell A, Cook R. Integration of spatial maps in pigeons. Anim Cogn. 2005; 8:7-16. [PubMed: 15221636]

Blanchard J, Decorte L, Noguès X, Micheau J. Characterization of cognition alteration across the course of the disease in app751 sl mice with parallel estimation of cerebral a $\beta \beta$ deposition. Behav Brain Res. 2009; 201(1):147-157. [PubMed: 19428628]

Bouton, ME. Signals for whether versus when an event will occur. In: Bouton, ME.; Fanselow, MS., editors. Learning, motivation, and cognition: the functional behaviorism of Robert C. Bolles. American Psychological Association; Washington, DC: 1997. p. 385-409.

Brown MF. Does a cognitive map guide choices in the radial-arm maze? J Exp Psychol Anim Behav Process. 1992; 18(1):56-66. [PubMed: 1578200]

Brown MF, Rish PA, VonCulin JE, Edberg JA. Spatial guidance of choice behavior in the radial-arm maze. J Exp Psychol Anim Behav Process. 1993; 19(3):195-214. [PubMed: 8340766]

Chamizo VD, Rodrigo T, Mackintosh NJ. Spatial integration with rats. Learn Behav. 2006; 34:348354. [PubMed: 17330524]

Cheng K, Shettleworth SJ, Huttenlocher J, Rieser JJ. Bayesian integration of spatial information. Psychol Bull. 2007; 133:625-637. [PubMed: 17592958]

Christensen DZ, Bayer TA, Wirths O. Intracellular a $\beta$ triggers neuron loss in the cholinergic system of the app/ps1ki mouse model of Alzheimer's disease. Neurobiol Aging. 2010; 31(7):1153-1163. [PubMed: 18771817]

Clayton NS, Dickinson A. Episodic-like memory during cache recovery by scrub jays. Nature. 1998; 395(6699):272-274. [PubMed: 9751053] 
Clayton NS, Dickinson A. Memory for the content of caches by scrub jays (Aphelocoma coerulescens). J Exp Psychol Anim Behav Process. 1999a; 25(1):82-91. [PubMed: 9987859]

Clayton NS, Dickinson A. Motivational control of caching behaviour in the scrub jay, Aphelocoma coerulescens. Anim Behav. 1999b; 57(2):435-444. [PubMed: 10049484]

Clayton NS, Dickinson A. Scrub jays (Aphelocoma coerulescens) remember the relative time of caching as well as the location and content of their caches. J Comp Psychol. 1999c; 113(4):403416. [PubMed: 10608564]

Clayton NS, Yu KS, Dickinson A. Scrub jays (Aphelocoma coerulescens) form integrated memories of the multiple features of caching episodes. J Exp Psychol Anim Behav Process. 2001; 27(1):17-29. [PubMed: 11199511]

Clayton NS, Bussey TJ, Dickinson A. Can animals recall the past and plan for the future? Nat Rev Neurosci. 2003a; 4(8):685-691. [PubMed: 12894243]

Clayton NS, Yu KS, Dickinson A. Interacting cache memories: evidence for flexible memory use by western scrub-jays (Aphelocoma californica). J Exp Psychol Anim Behav Process. 2003b; 29(1): 14-22. [PubMed: 12561130]

Crystal JD. Elements of episodic-like memory in animal models. Behav Process. 2009; 80(3):269-277.

Crystal JD. Episodic-like memory in animals. Behav Brain Res. 2010; 215:235-243. [PubMed: 20211205]

de Kort SR, Dickinson A, Clayton NS. Retrospective cognition by food-caching western scrub-jays. Learn Motiv. 2005; 36(2):159-176.

Dere E, Huston JP, De Souza Silva MA. Episodic-like memory in mice: Simultaneous assessment of object, place and temporal order memory. Brain Res Protoc. 2005; 16(1-3):10-19.

Egerhazi A, Berecz R, Bartok E, Degrell I. Automated neuropsychological test battery (cantab) in mild cognitive impairment and in Alzheimer's disease. Prog Neuropsychopharmacol Biol Psychiatry. 2007; 31(3):746-751. [PubMed: 17289240]

Eriksen JL, Janus CG. Plaques, tangles, and memory loss in mouse models of neurodegeneration. Behav Genet. 2007; 37(1):79-100. [PubMed: 17072762]

Feeney M, Roberts WA, Sherry D. Memory for what, where, and when in the black-capped chickadee. Anim Cogn. 2009; 12(6):767-777. [PubMed: 19466468]

Gallistel, CR. The organization of learning. MIT Press; Cambridge: 1990.

Hampton RR, Hampstead BM, Murray EA. Rhesus monkeys (Macaca mulatta) demonstrate robust memory for what and where, but not when, in an open-field test of memory. Learn Motiv. 2005; 36(2):245-259.

Hoffman ML, Beran MJ, Washburn DA. Memory for "What", "Where", and "When" Information in rhesus monkeys (Macaca mulatta). J Exp Psychol Anim Behav Process. 2009; 35(2):143-152. [PubMed: 19364223]

Hwang DY, Cho JS, Lee SH, Chae KR, Lim HJ, Min SH, Seo SJ, Song YS, Song CW, Paik SG, Sheen YY, Kim YK. Aberrant expressions of pathogenic phenotype in Alzheimer's diseased transgenic mice carrying nse-controlled appsw. Exp Neurol. 2004; 186(1):20-32. [PubMed: 14980807]

Jankowsky JL, Slunt HH, Gonzales V, Savonenko AV, Wen JC, Jenkins NA, Copeland NG, Younkin LH, Lester HA, Younkin SG, Borchelt DR. Persistent amyloidosis following suppression of a $\beta$ production in a transgenic model of Alzheimer disease. PLoS Med. 2005; 2(12):e355-e1333. [PubMed: 16279840]

Keri, RA.; Siegel, RE.; Donald, WP.; Arthur, PA.; Susan, EF.; Anne, ME.; Robert, TR. Transgenic and genetic animal models. In: Pfaff, DW.; Fahrbach, SE.; Etgen, AM.; Rubin, RT., editors. Hormones, brain and behavior. Academic Press; San Diego: 2009. p. 2673-2708.

Kessels RPC, Hobbel D, Postma A. Aging, context memory and binding: a comparison of "What, where and when" in youg and older adults. Int J Neurosci. 2007; 117(6):795-810. [PubMed: 17454244]

Le Moal S, Reymann JM, Thomas V, Cattenoz C, Lieury A, Allain H. Effect of normal aging and of Alzheimer's disease on episodic memory. Dement Geriatr Cogn Disord. 1997; 8(5):281-287. [PubMed: 9298629] 
Liscic RM, Storandt M, Cairns NJ, Morris JC. Clinical and psychometric distinction of frontotemporal and Alzheimer dementias. Arch Neurol. 2007; 64(4):535-540. [PubMed: 17420315]

Lovasic L, Bauschke H, Janus C. Working memory impairment in a transgenic amyloid precursor protein tgcrnd8 mouse model of Alzheimer's disease. Genes Brain Behav. 2005; 4(3):197-208. [PubMed: 15810906]

Maxwell MM. Rnai applications in therapy development for neurodegenerative disease. Curr Pharm Des. 2009; 15:3977-3991. [PubMed: 19751205]

Mazmanian DS, Roberts WA. Spatial memory in rats under restricted viewing conditions. Learn Motiv. 1983; 14(2):123-139.

Naqshbandi M, Feeney MC, McKenzie TLB, Roberts WA. Testing for episodic-like memory in rats in the absence of time of day cues: replication of Babb and Crystal. Behav Process. 2007; 74(2):217225 .

Nyberg L, McIntosh A, Cabeza R, Habib R, Houle S, Tulving E. General and specific brain regions involved in encoding and retrieval of events: what, where, and when. Proc Natl Acad Sci USA. 1996; 93:11280-11285. [PubMed: 8855347]

Oddo S, Caccamo A, Kitazawa M, Tseng BP, LaFerla FM. Amyloid deposition precedes tangle formation in a triple transgenic model of Alzheimer's disease. Neurobiol Aging. 2003a; 24(8): 1063-1070. [PubMed: 14643377]

Oddo S, Caccamo A, Shepherd JD, Murphy MP, Golde TE, Kayed R, Metherate R, Mattson MP, Akbari Y, LaFerla FM. Triple-transgenic model of Alzheimer's disease with plaques and tangles: intracellular $\mathrm{a} \beta$ and synaptic dysfunction. Neuron. 2003b; 39(3):409-421. [PubMed: 12895417]

Olton DS, Collison C. Intramaze cues and odor trails fail to direct choice behavior on a elevated maze. Anim Learn Behav. 1979; 7(2):221-223.

Roberts, WA. Principles of animal cognition. McGraw-Hill; Boston: 1998.

Roberts WA, Feeney MC, MacPherson K, Petter M, McMillan N, Musolino E. Episodic-like memory in rats: Is it based on when or how long ago? Science. 2008; 320(5872):113-115. [PubMed: 18388296]

Savonenko A, Xu GM, Melnikova T, Morton JL, Gonzales V, Wong MPF, Price DL, Tang F, Markowska AL, Borchelt DR. Episodic-like memory deficits in the appswe/ps1de9 mouse model of Alzheimer's disease: relationships to $\beta$-amyloid deposition and neurotransmitter abnormalities. Neurobiol Dis. 2005; 18:602-617. [PubMed: 15755686]

Shettleworth, SJ. Cognition, evolutoin, and behavior. Oxford University Press; New York: 1998.

Singer RA, Zentall TR. Pigeons learn to answer the question 'where did you just peck?' and can report peck location when unexpectedly asked. Learn Behav. 2007; 35(3):184-189. [PubMed: 17918424]

Suzuki S, Augerinos G, Black AH. Stimulus control of spatial behavior on the eight-arm maze in rats. Learn Motiv. 1980; 11(1):1-18.

Tulving, E. Episodic and semantic memory. In: Tulving, E.; Donaldson, W., editors. Organization of memory. Academic Press; New York: 1972. p. 381-403.

Tulving, E. Oxford psychology series no. 2. Oxford University Press; New York: 1983. Elements of episodic memory.

Tulving E. How many memory systems are there? Am Psychol. 1985; 40(4):385-398.

Tulving E. Episodic memory: from mind to brain. Annu Rev Psychol. 2002; 53(1):1-25. [PubMed: 11752477]

Tulving E, Markowitsch HJ. Episodic and declarative memory: role of the hippocampus. Hippocampus. 1998; 8(3):198-204. [PubMed: 9662134]

Ueberham U, Zobiak B, Ueberham E, Brückner MK, Boriss H, Arendt T. Differentially expressed cortical genes contribute to perivascular deposition in transgenic mice with inducible neuronspecific expression of tgf- $\beta$ 1. Int J Dev Neurosci. 2006; 24(2-3):177-186. [PubMed: 16386398]

Volianskis A, Køstner R, Mølgaard M, Hass S, Jensen MS. Episodic memory deficits are not related to altered glutamatergic synaptic transmission and plasticity in the cal hippocampus of the appswe/ ps1 $\Delta$ e9-deleted transgenic mice model of $\beta$-amyloidosis. Neurobiol Aging. 2010; 31(7):11731187. [PubMed: 18790549] 
Wallace DG, Hines DJ, Pellis SM, Whishaw IQ. Vestibular information is required for dead reckoning in the rat. J Neurosci. 2002; 22(22):10009-10017. [PubMed: 12427858]

Wallace DG, Martin MM, Winter SS. Fractionating dead reckoning: role of the compass, odometer, logbook, and home base establishment in spatial orientation. Naturwissenschaften. 2008; 95(11): 1011-1026. [PubMed: 18553065]

Watanabe T, Yamagata N, Takasaki K, Sano K, Hayakawa K, Katsurabayashi S, Egashira N, Mishima $\mathrm{K}$, Iwasaki K, Fujiwara M. Decreased acetylcholine release is correlated to memory impairment in the tg2576 transgenic mouse model of Alzheimer's disease. Brain Res. 2009; 1249:222-228. [PubMed: 18996097]

Yoshiyama Y, Higuchi M, Zhang B, Huang S-M, Iwata N, Saido Takaomi C, Maeda J, Suhara T, Trojanowski JQ, Lee VMY. Synapse loss and microglial activation precede tangles in a p301 s tauopathy mouse model. Neuron. 2007; 53(3):337-351. [PubMed: 17270732]

Zentall TR. Animals may not be stuck in time. Learn Motiv. 2005; 36(2):208-225.

Zentall TR. Mental time travel in animals: a challenging question. Behav Process. 2006; 72(2):173183.

Zentall TR, Clement TS, Bhatt RS, Allen J. Episodic-like memory in pigeons. Psychon Bull Rev. 2001; 8(4):685-690. [PubMed: 11848586]

Zentall TR, Singer RA, Stagner JP. Episodic-like memory: pigeons can report location pecked when unexpectedly asked. Behav Process. 2008; 79(2):93-98.

Zhou W, Crystal JD. Evidence for remembering when events occurred in a rodent model of episodic memory. Proc Natl Acad Sci. 2009; 106(23):9525-9529. [PubMed: 19458264]

Zinkivskay A, Nazir F, Smulders T. What-where-when memory in magpies (Pica pica). Anim Cogn. 2009; 12(1):119-125. [PubMed: 18670793] 


\section{Morning \\ First Helpings}
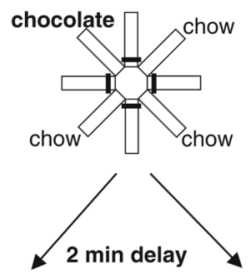

Retrieval Cue
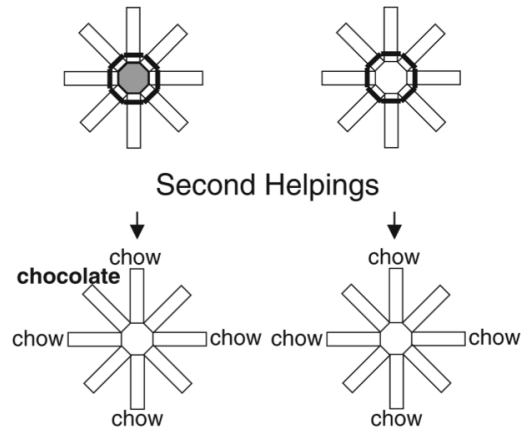

Afternoon

First Helpings

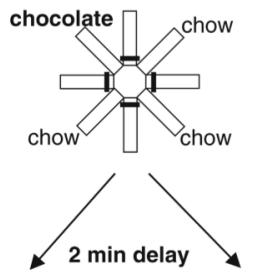

Retrieval Cue
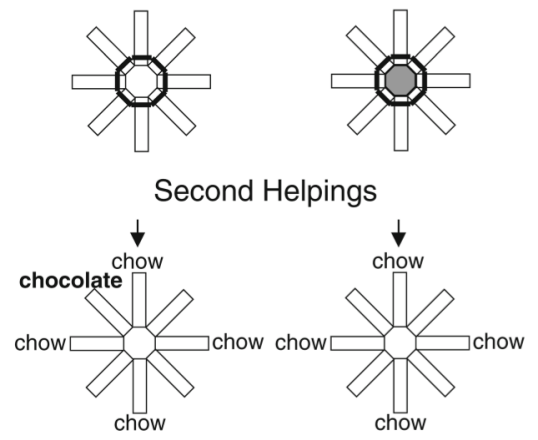

- closed door $\bigcirc$ chocolate in hub

Fig. 1.

Schematic representation of experimental design showing topographical views of the maze. The morning or afternoon was randomly selected for presentation of first helpings of food (encoding phase) and second helpings (memory-assessment phase). The figure shows an example of the accessible arms and flavors in encoding and the corresponding memoryassessment phases that would occur after a 2-min retention interval. The presence or absence of chocolate pellets in the central hub immediately prior to memory assessment was needed to decode the replenishment of chocolate at second helpings. In the replenishment conditions, chocolate replenished at the location that recently delivered chocolate, which was predicted by the presence or absence of food (e.g., presence of chocolate in the central hub immediately prior to second helpings memory assessment in the morning but absence of chocolate in the hub in the afternoon); these contingencies were reversed in the nonreplenishment conditions. These conditions were counterbalanced across rats (not shown). For each rat, 1 session (i.e., first helpings, retrieval cue, and second helpings) was conducted per day. The same arms were used to illustrate morning and afternoon sessions in the figure to facilitate inspection of presence and absence of chow and chocolate, but these arms were randomly selected in each session for each rat 


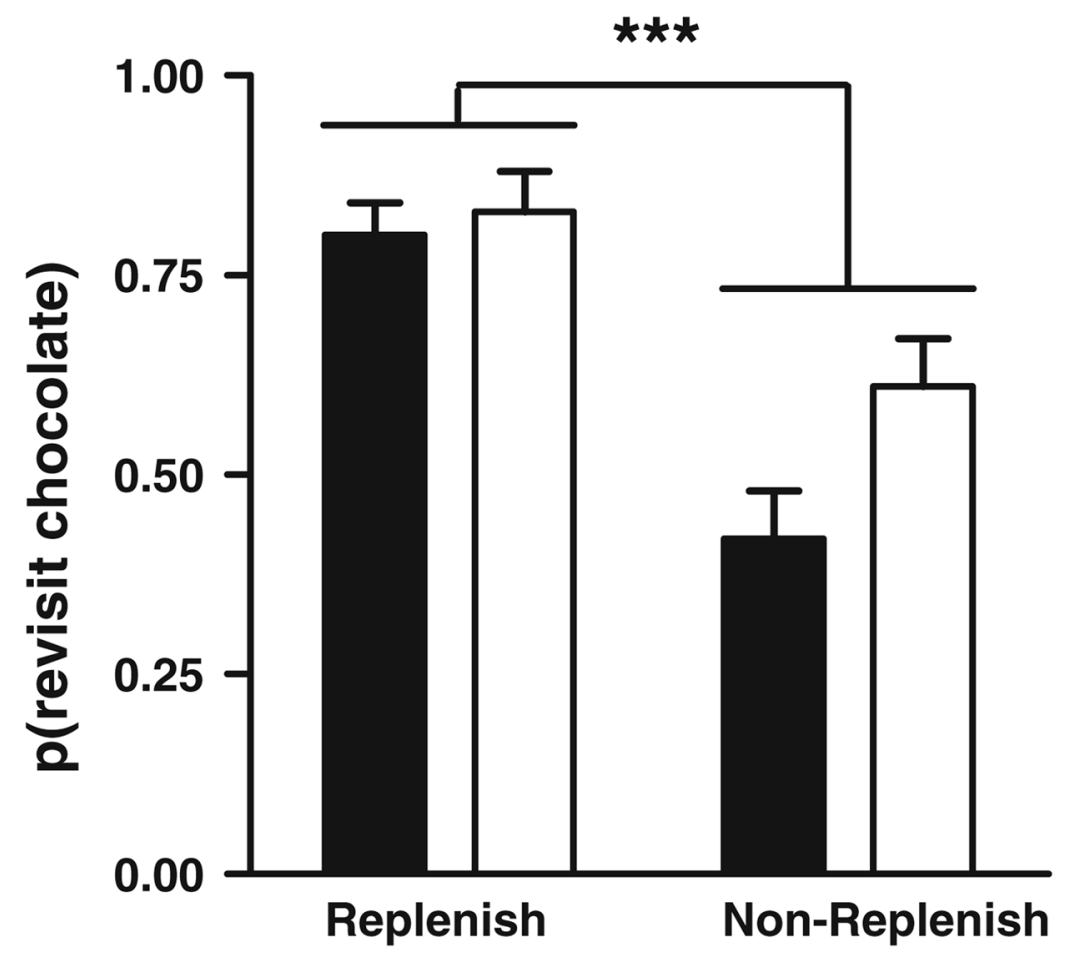

Food in Hub

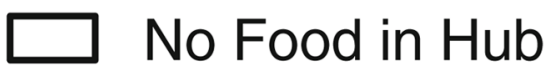

Fig. 2.

Rats preferentially revisit the chocolate location when it is about to replenish. The probability of a revisit to the chocolate location in the first four choices of a test phase is shown for replenishment and non-replenishment conditions; replenishment and nonreplenishment conditions were presented in random order. The presence or absence of food in the hub, immediately prior to memory assessment, served as a cue that could be used to predict the replenishment or non-replenishment of chocolate. Error bars represent 1 SEM. $* * * p<0.001$ difference between replenishment and non-replenishment conditions 


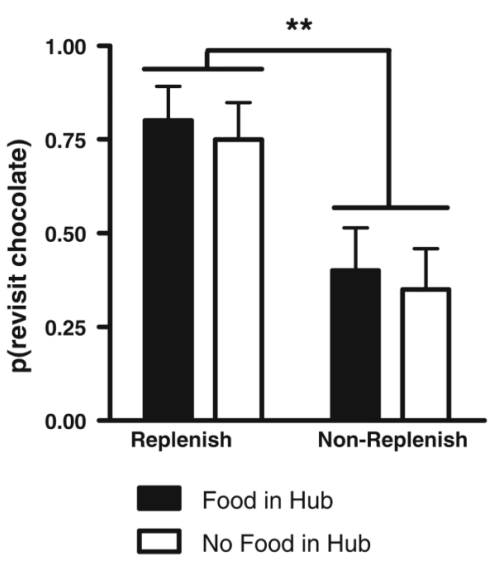

Fig. 3.

Rats preferentially revisit the chocolate location when it is about to replenish when the retention interval was approximately $1 \mathrm{~h}$. The probability of a revisit to the chocolate location in the first four choices of a test phase is shown for first replenishment and first non-replenishment conditions. Each condition was tested once, in random order. Error bars represent 1 SEM. $* * p=0.009$ difference between replenishment and non-replenishment conditions 

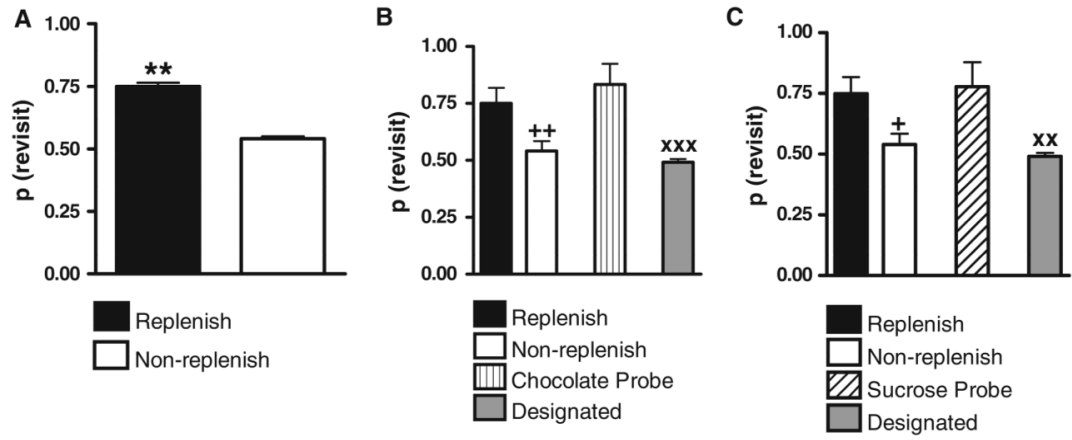

Fig. 4.

a Rats preferentially revisit the chocolate location when it is about to replenish. These data come from the last ten sessions of mixed testing of replenishment and non-replenishment conditions in Maze 1 and are referred to as the mixed baseline. $* * p=0.02$ difference between replenishment and non-replenishment conditions. $\mathbf{b}$ In the chocolate probe, rats revisited the corresponding chocolate location in Maze 2 at a higher rate when chocolate was unexpectedly replenished compared with the non-replenishment mixed baseline in Maze 1 (labeled Replenish and Non-replenish) and designated baseline in Maze 2 (labeled Designated). The probability of revisiting corresponding chocolate location in Maze 2 is labeled Chocolate Probe. The mixed baseline is reproduced from a to facilitate comparison with the chocolate probe. ${ }^{++} p=0.005$ difference between chocolate probe and nonreplenishment mixed baseline; ${ }^{\mathrm{xx}} p=0.001$ difference between chocolate probe and designated baseline. $\mathbf{c}$ In the sucrose probe, rats revisited the corresponding sucrose location in Maze 2 when sucrose was unexpectedly replenished compared with the nonreplenishment mixed baseline in Maze 1 and designated baseline in Maze $2 .{ }^{+} p=0.03$ (onetailed) difference between sucrose probe and non-replenishment mixed baseline; ${ }^{\mathrm{xx}} p=0.01$ difference between sucrose probe and designated baseline. a-c Error bars represent 1 SEM 